ISSN 1112-9867

http://www.jfas.info

\title{
STUDY AND NUMERICAL SIMULATION OF SOLAR SYSTEM FOR AIR HEATING
}

\author{
M. Ghodbane ${ }^{1, *}$, B. Boumeddane ${ }^{1}$, N. Moummi ${ }^{2}$, S. Largot ${ }^{3}$, H. Berkane ${ }^{3}$ \\ ${ }^{1}$ Faculty of Technology, University of Saad DAHLAB, 1 Blida, Algeria \\ ${ }^{2}$ Laboratory of Mechanical Engineering, University of Mohamed KHIDER, Biskra, Algeria \\ ${ }^{3}$ Faculty of Technology, University of Echahid HAMMA Lakhder, El Oued, Algeria
}

Received: 04 September 2015 / Accepted: 16 December 2015 / Published online: 01 January 2016

\begin{abstract}
The use of solar energy in sunny countries, is an effective outil for compensate the lack in the energy, their benefits are not related only to its economic benefits but especially for the environmental protection, so we must find solutions to the problems of pollution. This work is a theoretical study of a solar flat plate collector; air is used as the heat transfer fluid. In this study, we established in first step calculation of solar radiation in various sites in Algeria (Adrar, El Oued, Bechar, Biskra and Tamanrasset). The second step is the parameters influence study of the sites and climate on the performance of our collector. The results obtained are encouraging for the use of this type in the heating in the winter; also it can be used in different kinds of drying.
\end{abstract}

Keywords: thermal energy; solar collector plane; air; simulation; outlet temperature.

Author Correspondence, e-mail: ghodbanemokhtar39@yahoo.com

doi: http://dx.doi.org/10.4314/jfas.v8i1.3 


\section{INTRODUCTION}

Les ressources énergétiques mondiales peuvent être classées en [1] :

Ressources fossiles (non renouvelables) qui se sont accumulées dans l'écorce terrestre au cours des ères géologiques, du fait de processus biologiques ou physico-chimiques.

Ressources renouvelables (solaire, éolien et géothermie). L'énergie solaire est la plus dominante de toutes les énergies renouvelables, elle est à l'origine de la quasi-totalité des sources d'énergies utilisées par l'homme [1-2].

Actuellement, la conservation des ressources énergétiques est devenue une priorité à l'échelle planétaire, d'autre part, vue la demande vertigineuse en matière d'énergie, ce qui a poussé les spécialistes à trouver de nouvelles techniques, telles que les énergies solaire. L'utilisation de l'énergie solaire commence par sa conversion. Il y a deux classes de système de conversion:

- La conversion de l'énergie solaire reçue par les collecteurs solaire en énergie thermique, elle est transformée en énergie calorifique.

- La conversion électrique (système photovoltaïque), où l'énergie solaire collectée est transformée en électricité.

Les capteurs solaires plans à air, qui sont l'objet de notre étude, transforment l'énergie solaire incidente en énergie thermique extraite par l'air en écoulement dans le capteur. Cette énergie et ensuite utilisée dans diverses applications, à savoir; le chauffage, la réfrigération, le séchage, ...etc.

Dans les précédents articles [3-4], nous avons parlé de l'exploitation de l'énergie solaire dans la production de vapeur en utilisant un concentrateur cylindro-parabolique. Nous avons acquis d'excellents résultats, qui encouragent l'exploitation de ce type des concentrateurs en Algérie.

Dans cet article, nous uilisons le capteur solaire plan pour le chauffage de l'air dans le mois de Janvier. On précède sur quelques notions fondamentales d'astronomie qui donnent des informations sur le rayonnement global reçu par le sol et l'évaluation de la température d'entrée et de sortie en fonction des paramètres climatiques et géographiques pour les lieux sélectionnés pour mener l'étude. L'étude a été menée sur les villes suivantes : Adrar, Béchar, Biskra, El Oued et Tamanrasset, les cinq sites sont des villes sahariennes qui se situent au sud Algérien. Nous avons utilisé Matlab comme langage de programmation. 


\section{METHODOLOGIE}

\subsection{Capteur Solaire Plan Comme Convertisseur Solaire/Thermique}

Les systèmes de conversion d'énergie solaire en énergie thermique en utilisant le capteur solaire plan sont les plus anciens, les plus avancés et les plus économiques $[1,2,5]$. Le capteur solaire est un échangeur de chaleur, il est composé de surface noire (absorbeur) qui transforme le rayonnement solaire en chaleur et le transmet au fluide caloporteur (air ou eau) [6]. La capacité de transformation de l'irradiation solaire en chaleur d'une installation dépend du besoin et l'utilité qu'on veut donner à la production de cette chaleur, suivant les procédés de transformation, on peut classifier les systèmes solaires:

- $\quad$ Basse et moyenne température $\left(\leq 100^{\circ} \mathrm{C}\right)$ : (Chauffage central, Eau chaude sanitaire, Réfrigération, Dessalement de l'eau de mer, Séchage des fruits, des légumes, ...etc.).

- $\quad$ Haute température $\left(>100^{\circ} \mathrm{C}\right)$ : (Procédés thermiques, Stérilisation.)

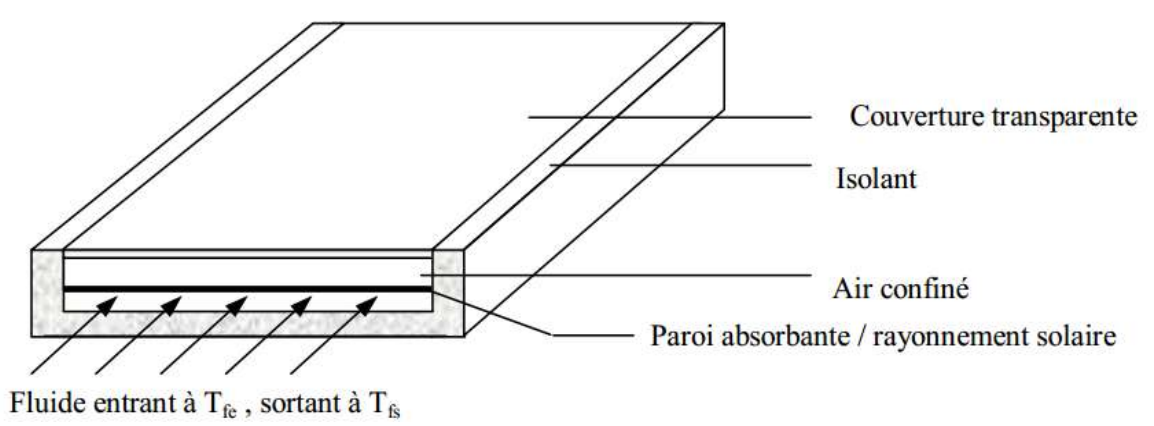

Fig.1. Schéma d'un capteur solaire plan à air [7].

\subsection{Rayonnement global reçu par une surface inclinée}

Ces composantes peuvent être mesurées directement par différents instruments de mesure ou bien elles peuvent être estimées par des modèles analytiques qui sont établis après plusieurs mesures expérimentales.

Le rayonnement solaire global « $\mathrm{G} »$ arrivant sur une surface orientée au sud d'inclinaison $« \beta »$ (Fig. 2) est formé de rayonnement direct et de rayonnement diffus $[1,2,5,6]$. Ces composantes peuvent être mesurées directement par différents instruments de mesure ou bien elles peuvent être estimées par des modèles analytiques qui sont établis après plusieurs mesures expérimentales. Parmi ces modèles on a présenté le modèle de PERRIN DE 
BRICHAMBAUT, ce modèle donne de bons résultats losqui’il est appliqué en Algérie.

Pour cela on a élaboré un organigramme de calcul du rayonnement solaire sur un plan incliné où on a adopté une inclinaison égale à la latitude du site pour notre capteur et nous avons calculé les insolations pour le 06 Janvier 2011. Il peut estimer le rayonnement solaire global à n'importe quel instant et dans n'importe quel endroit. On a fait l'application des formules de PERRIN DE BRICHAMBAUT concernant le rayonnement solaire global. En utilisant le logiciel Matlab on a pu estimer l'énergie solaire rayonnante pour une journée précise de l'année et pour des conditions climatiques données. Le modèle de PERRIN DE BRICHAMBAUT donnant le rayonnement global est présenté par la formule suivante [1-2] :

$$
G=I_{d n}+D_{C}(\beta)+D_{S}(\beta)
$$

Où $I_{d n}$ : est le rayonnement normal reçu par une surface d'inclinaison « $\beta »[2,8], D_{c}(\beta)$ : est le rayonnement diffus provenant du ciel, reçu par une surface d'inclinaison « $\beta$ »et $D_{s}(\beta)$ : est le rayonnement diffus provenant du sol capté par une surface horizontale [W/m²] [9].

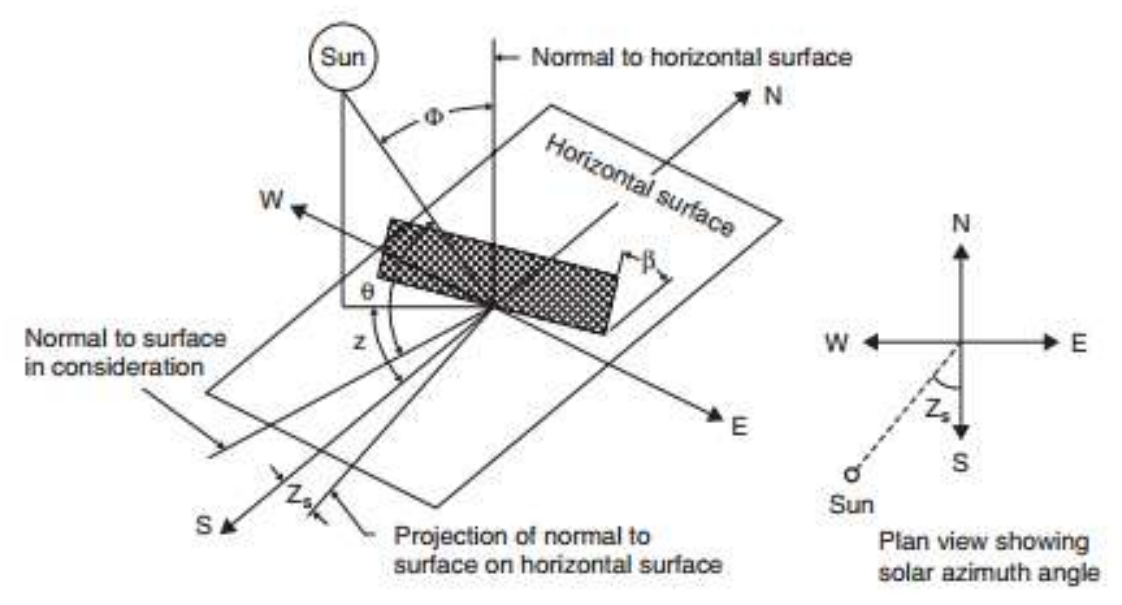

Fig.2. Caractéristiques géométriques d'un capteur plan [1].

L'irradiation directe reçu par une surface d'inclinaison « $\beta$ » est exprimée par la relation:

$$
\mathrm{I}_{\mathrm{dn}}=\mathrm{I}_{\mathrm{d}} \cos (\mathrm{u})
$$


Avec u : est l'angle que fait la normale à la surface de captation avec la direction des rayons solaires et $I_{d}$ : est le rayonnement direct provenant du ciel $\left[\mathrm{W} / \mathrm{m}^{2}\right]$, on utilise généralement les formules semi-empiriques suivantes [9]:

- Ciel très clair (CTC) :

$$
\mathrm{I}_{\mathrm{d}}=1210 \exp \left(-\frac{1}{6 \sin (h+1)}\right)
$$

h est la hauteur du soleil $\left[{ }^{\circ}\right]$.

- Conditions normales de ciel clair (CNCC) :

$$
\mathrm{I}_{\mathrm{d}}=1230 \exp \left(-\frac{1}{3.8 \sin (h+1.6)}\right)
$$

- Ciel clair pollué (CCP):

$$
\mathrm{I}_{\mathrm{d}}=1260 \exp \left(-\frac{1}{2.3 \sin (h+3)}\right)
$$

h est la hauteur du soleil étant en degrés.

$D_{c}(\beta)$ : est le rayonnement diffus provenant du ciel, reçu par une surface d'inclinaison « $\beta$ » [9].

$$
D_{c}(\beta)=\frac{1+\cos (\beta)}{2} D_{h}
$$

$\mathrm{D}_{\mathrm{h}}$ : est le rayonnement diffus provenant du ciel intercepté par une surface horizontale $\left[\mathrm{W} / \mathrm{m}^{2}\right][9]$.

- Ciel très clair (CTC) :

$$
D_{h}=\frac{3}{4} 125 \sin (h)^{0.4}
$$

- Conditions normales de ciel clair (CNCC) :

$$
D_{h}=125 \sin (h)^{0.4}
$$

- Ciel clair pollué (CCP):

$$
D_{h}=\frac{4}{3} 125 \sin (h)^{0.4}
$$


$D_{s}(\beta)$ : est le rayonnement diffus provenant du sol capté par une surface horizontale $\left[\mathrm{W} / \mathrm{m}^{2}\right]$ [9].

$$
D_{S}(\beta)=\alpha_{\text {sol }} \frac{1-\cos (\beta)}{2}\left(I_{d} \sin (h)+D_{h}\right)
$$

Avec $\left(\alpha_{\text {sol }}\right)$ est la réflectivité ou albédo du sol, il dépend de la nature de celui-ci. Quelques valeurs moyennes sont résumées dans le tableau (1).

Tableau 1. Quelques valeurs de l'albédo en fonction de la nature du Sol [10].

\begin{tabular}{cc}
\hline NATURE DU SOL & ALBEDO \\
\hline Sol enneigé & 0.70 \\
Sol recouvert de feuilles mortes & 0.30 \\
Herbe verte & 0.26 \\
Forêt en automne ou champs dorés & 0.26 \\
Galets de pierres blanches & 020 \\
Herbe sèche & 0.20 \\
Sol argileux & 0.17 \\
Forêt en hiver (arbres conifères sans neige) & 0.07 \\
Plan d'eau (soleil haut $\mathrm{h}>30^{\circ}$ ) à & 0.07 \\
\hline
\end{tabular}

\subsection{Calcul d'efficacité}

L'efficacité du collecteur est le rapport entre la quantité de la chaleur nécessaire pour chauffer l'air et la quantité de la chaleur reçue par l'absorbeur [2, 11-12], il est donné par l'expréssion suivante [2, 13-15]:

$$
\eta=\frac{\mathrm{Q}_{\mathrm{u}}}{\mathrm{G}_{\mathrm{A}} \mathrm{c}}=\frac{\rho \cdot \mathrm{C}_{\mathrm{p}} \cdot \mathrm{Q}_{\mathrm{v}} \cdot\left(\mathrm{T}_{\mathrm{S}}-\mathrm{T}_{\mathrm{e}}\right)}{\mathrm{G}_{\mathrm{A}} \mathrm{A}_{\mathrm{c}}}
$$

Où $\mathrm{Q}_{\mathrm{u}}$ : est la puissance utile pour chauffer l'air [W], $\mathrm{A}_{\mathrm{c}}$ : est surface de captation de l'insolateur plan à air $\left[\mathrm{m}^{2}\right], \mathrm{C}_{\mathrm{p}}$ : est la chaleur spécifique de l'air $\left[\mathrm{J} / \mathrm{Kg} .{ }^{\circ} \mathrm{C}\right], \mathrm{Q}_{\mathrm{v}}$ : est le débit volumique de l'air $\left[\mathrm{m}^{3} \cdot \mathrm{h}^{-1} \cdot \mathrm{m}^{-2}\right], \mathrm{T}_{\mathrm{e}}$ : est La température d'entrée de l'air $\left[{ }^{\circ} \mathrm{C}\right]$ et $\mathrm{T}_{\mathrm{S}}$ est la température de la sortie de l'air $\left[{ }^{\circ} \mathrm{C}\right]$.

$\rho$ est la densité de l'air à l'instant $(\mathrm{t})\left[\mathrm{Kg} / \mathrm{m}^{3}\right]$, elle peut être calculée comme suit: 


$$
\rho=\rho_{0} \frac{273}{273+T_{m}} \frac{\mathrm{P}(\mathrm{z})}{\mathrm{P}_{0}}
$$

Où $\mathrm{T}_{\mathrm{m}}$ est la température mesurée au niveau des tubes absorbeur au cours de l'écoulement de l'air $\left[{ }^{\circ} \mathrm{C}\right]$.

La notion $(\mathrm{P}(\mathrm{z}) / \mathrm{P} 0)$ est la correction d'altitude $(\mathrm{z})$. Pour des altitudes inférieures à $3.5[\mathrm{~km}]$, on l'admet cela [14]:

$$
\frac{\mathrm{P}(\mathrm{z})}{\mathrm{P}_{0}}=(0.89)^{z}
$$

Avec $\mathrm{z}$ est l'altitude exprimée en [km].

\subsection{Modèle de la température d'entrée}

L'évolution de la température d'entrée, en fonctin du temps $T_{\mathrm{e}}(\mathrm{t})$, pendant un jour ensoleillé suit une fonction sinusoïdale de la température moyenne; ce dernier est moitié de la somme entre la température maximale $T_{\max }$ et minimale $T_{\min }$ du jour correspondant $[14,16]$ :

$$
\mathrm{T}_{\mathrm{e}}(\mathrm{t})=\left[\frac{\mathrm{T}_{\max }+\mathrm{T}_{\min }}{2}\right]-\left[\frac{\mathrm{T}_{\max }+\mathrm{T}_{\min }}{2}\right] \cos \left(\frac{2 \pi}{\Delta \mathrm{t}}\right) \mathrm{t}
$$

Avec $(\Delta t)$ est la durée du jour, $\mathrm{t}$ est le temps solaire vrai [heure], $\mathrm{T}_{\max }$ est la température maximal de l'ambiant $\left[{ }^{\circ} \mathrm{C}\right]$ et $\mathrm{T}_{\min }$ est la température minimal de l'ambiant $\left[{ }^{\circ} \mathrm{C}\right]$.

\subsection{Calcul de la température de sortie}

De l'équation (11) et pour calculer la température de sortie $T_{S}$ avec un rendement de capteur égal à $60 \%$ pour $\mathrm{Q}_{\mathrm{v}}=20\left[\mathrm{~m}^{3} / \mathrm{h} \cdot \mathrm{m}^{2}\right]$ et $73 \%$ pour $\mathrm{Q}_{\mathrm{v}}=35\left[\mathrm{~m}^{3} / \mathrm{h} \cdot \mathrm{m}^{2}\right][14,16]$, il est possible de déduire l'expression de la température de sortie du collecteur. Des calculs ont été faits pour deux - des valeurs d'écoulement comme suit:

- pour débit d'air $\mathrm{Q}_{\mathrm{v}}=20\left[\mathrm{~m}^{3} / \mathrm{h} \cdot \mathrm{m}^{2}\right][14,16]$.

$$
\mathrm{T}_{\mathrm{S}}=\left(\frac{102.7 \mathrm{G}}{\mathrm{P}}\right)+\mathrm{T}_{\mathrm{e}}
$$

- pour un débit d'air $\mathrm{Q}_{\mathrm{v}}=35\left[\mathrm{~m}^{3} / \mathrm{h} \cdot \mathrm{m}^{2}\right][14,16]$.

$$
\mathrm{T}_{\mathrm{S}}=\left(\frac{71.4 \mathrm{G}}{\mathrm{P}}\right)+\mathrm{T}_{\mathrm{e}}
$$




\section{DONNEES GEOGRAPHIQUES CLIMATIQUES}

Nous avons sélectionné cinq lieux afin de mener l'étude. Les sites sont: Adrar, Béchar, Biskra, El Oued et Tamanrasset. Le tableau (2) montre les caractéristiques géographiques de chaque site (Les coordonnées des sites).

Tableau 2. Les données géographiques pour les cinq sites.

\begin{tabular}{ccccc}
\hline La ville & latitude $\left(^{\circ}\right)$ & longitude $\left(^{\circ}\right)$ & $\mathbf{z}(\mathbf{m})$ & Albédo \\
\hline Adrar & 27,53 & $-0,17$ & 264 & 0,2 \\
Béchar & 31,38 & $-2,15$ & 806 & 0,35 \\
Biskra & 34,48 & $+5,44$ & 81 & 0,3 \\
El Oued & 33,5 & $+6,8$ & 70 & 0,3 \\
Tamanrasset & 22,47 & $+5,31$ & 1348 & 0,35 \\
\hline
\end{tabular}

Ce tableau représente les données météorologiques pour la journée 01/06/2011 (saison d'hiver) pour les cinq sites. Le tableau contient la température minimale, la température maximale, la hauteur de la région concernée et le type de ciel pour chaque lieu [17].

Tableau 3. Les données climatiques pour les cinq sites.

\begin{tabular}{cccc}
\hline région & $\mathbf{t}_{\min }\left({ }^{\circ} \mathbf{c}\right)$ & $\mathbf{t}_{\max }\left({ }^{\circ} \mathbf{c}\right)$ & type de ciel \\
\hline Adrar & 6 & 23 & CTC \\
Béchar & 1 & 20 & CTC \\
Biskra & 4 & 18 & CTC \\
El Oued & 4 & 18 & CTC \\
Tamanrasset & 3 & 20 & CTC \\
\hline
\end{tabular}

\section{RESULTATS ET INTERPRETATION}

Dans cette partie nous allons présenter l'ensemble des résultats issus de la simulation numérique. Cette étude illustre l'énorme potentiel qui abonde dans notre pays dans le domaine de l'énergie solaire. Cette étude nous a donnés une simple estimation sur la température de 
l'air obtenue à l'aide de convertisseur solaire «capteur solaire plan » pour la journée de 06/01/2011.

\subsection{Moyenne mensuelle et annuelle de l'irradiation globale}

Le gisement solaire est exprimé par les sommes annuelles du rayonnement solaire global sur un plan horizontal. L'Algérie est le premier pays d'Afrique par sa superficie, plus des quatre cinquièmes du leur territoire sont désertiques et possède une source énergétique très importante de part de sa situation géographique. La durée annuelle du rayonnement solaire sur la quasi-totalité du territoire national dépasse 2000 heures, où peut atteindre les 3900 heures (hauts plateaux et Sahara). La figure (3) montre la moyenne annuelle de l'irradiation globale reçue sur une surface horizontale, période 1992-2002 dans le territoire national.

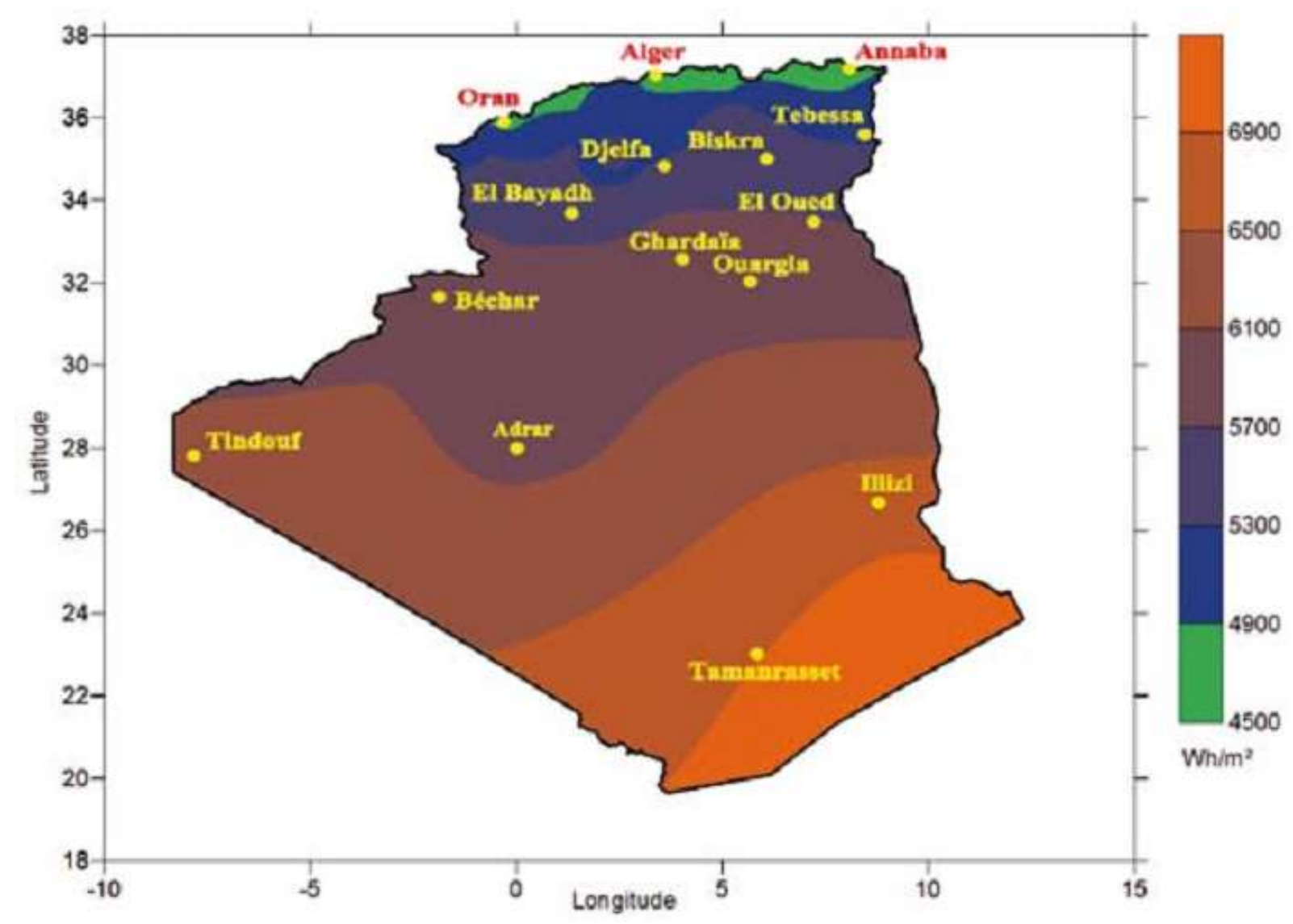

Fig.3. Moyenne annuelle de l'irradiation globale reçue sur une surface horizontale, période 1992-2002 [Wh/m²] [19]. 
Le potentiel énergétique solaire reçu quotidiennement sur une surface horizontale de $1 \mathrm{~m}^{2}$ est de l'ordre de $5[\mathrm{KWh}$ ] sur la majeure partie du territoire national, où nous trouvons que l'énergie moyenne reçue au niveau de la région côtière près de $1700\left[\mathrm{KWh} / \mathrm{m}^{2} / \mathrm{an}\right], 1900$ $\left[\mathrm{KWh} / \mathrm{m}^{2} / \mathrm{an}\right]$ aux hauts plateaux, et $2650\left[\mathrm{KWh} / \mathrm{m}^{2} / \mathrm{an}\right]$ au Sud du pays [18].

La figure (4) montre la moyenne mensuelle de l'irradiation globale reçue sur une surface horizontale, période 1992-2002 dans le territoire national.

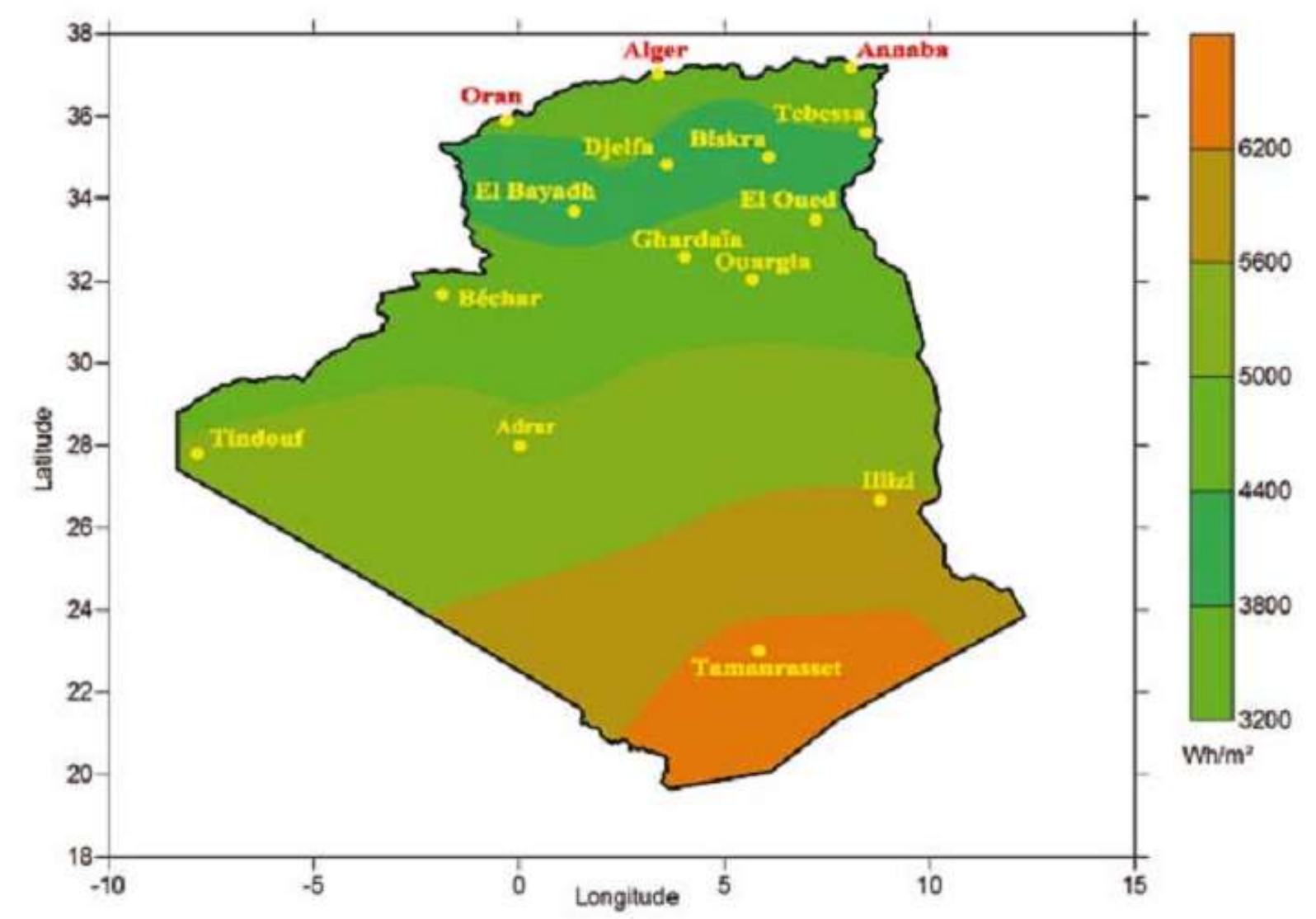

Fig.4. Moyenne mensuelle de l'irradiation globale reçue sur une surface horizontale, période 1992-2002 [Wh/m²] [19].

La figure (5) représente les valeurs du rayonnement global mensuel reçu sur un plan horizontal obtenues pour les cinq sites. 


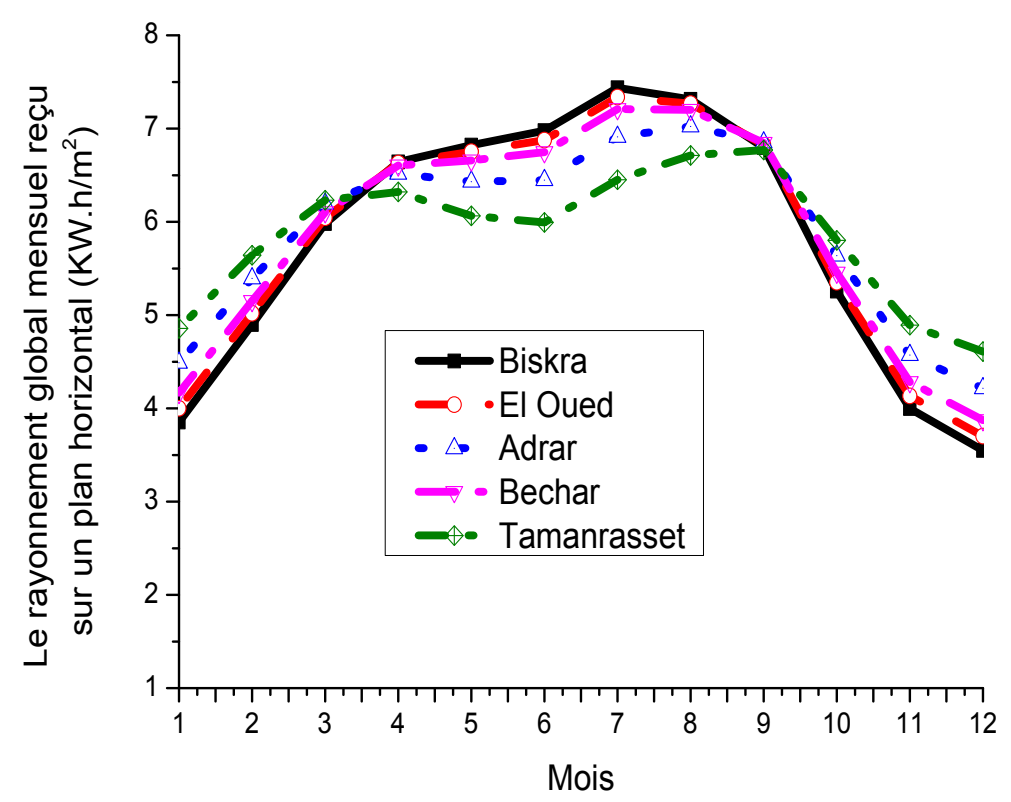

Fig.5. Le rayonnement global mensuel reçu sur un plan horizontal $\left[\mathrm{KW} \cdot \mathrm{h} / \mathrm{m}^{2}\right]$.

Nous remarquons que la quantité d'éclairement extérieur global reçue par une surface horizontale diffère d'un mois à l'autre. Cet éclairement doit être utilisé de manière intelligente et durable pour pouvoir répondre aux besoins qualitatifs et quantitatifs des énergies en termes d'électricité, de chauffage, de climatisation...etc.

\subsection{Le rayonnement global}

L'énergie solaire est en faite de rayons électromagnétiques qui composent le rayonnement solaire. Ce rayonnement est la seule source d'énergie externe pour l'atmosphère. Il se propage à la vitesse de la lumière (c), il prend 499 secondes pour atteindre la Terre [20]. La plus grande partie de l'énergie solaire est cependant rayonnée dans les domaines ultraviolet, visible et proche de l'infrarouge.

La quantité de radiation solaire reçue sur une surface unité perpendiculaire aux rayons solaires et pour la distance terre-soleil est appelé constante solaire, cette constante solaire a été estimée à $1366\left[\mathrm{~W} / \mathrm{m}^{2}\right]$ selon la norme ASTM E490-00a [21], et égal à $1367\left[\mathrm{~W} / \mathrm{m}^{2}\right]$ selon le centre radiométrique mondial de Davos (Suisse) [20].

La performance, des capteurs solaires, a un lien direct avec le flux solaire incident en fonction du temps, c'est à dire en fonction de la position du soleil par rapport à la terre. 
L'ensemble des résultats numériques obtenus, qui donnent l'évolution du rayonnement solaire sur un plan incliné en fonction des conditions climatiques et situation géographique de chaque zone pour la journée de simulation est représenté dans la figure (6). Les courbes montrent l'évaluation du rayonnement solaire global «G» $\left[\mathrm{W} / \mathrm{m}^{2}\right]$ incident sur le capteur dans les cinq sites en fonction du temps solaire vrai [heure] où on a supposé que le ciel est très clair. Ce fort rayonnement solaire doit être utilisé de manière intelligente et durable pour fournir des besoins quantitatifs et qualitatifs de l'énergie quotidienne.

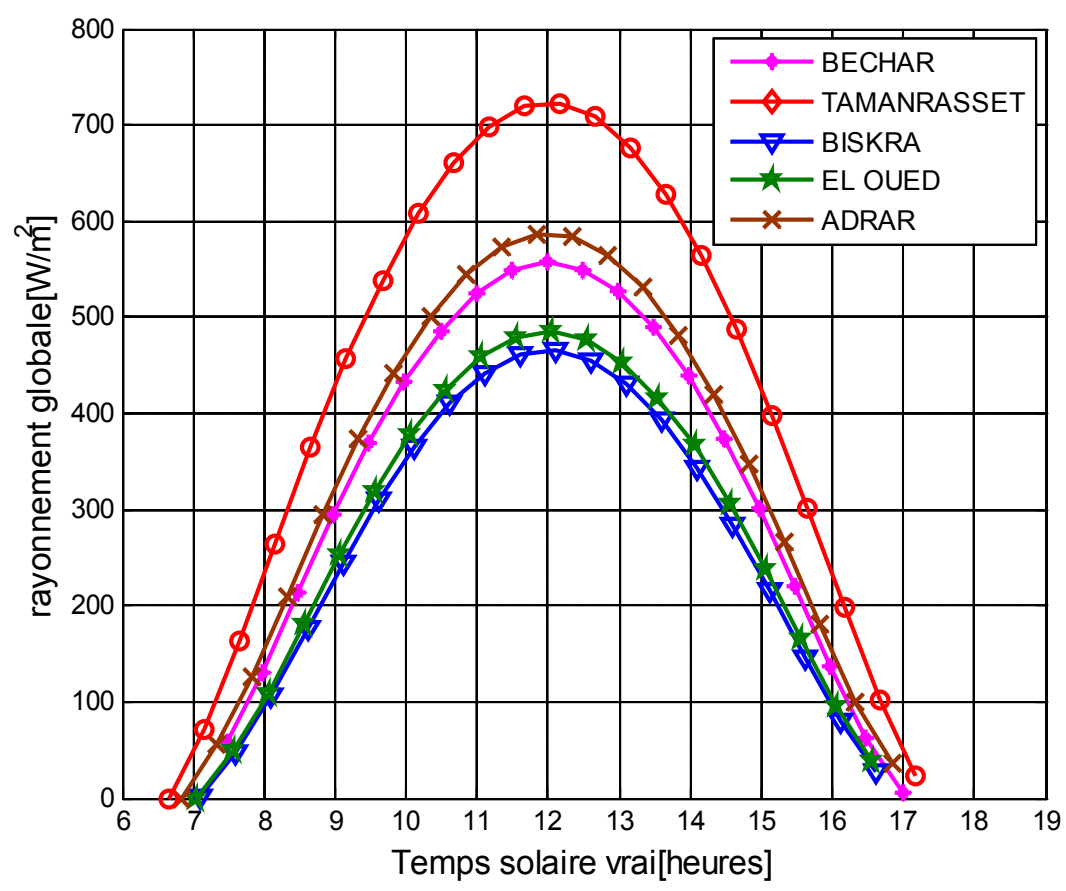

Fig.6. Le rayonnement solaire global sur un plan incliné pour les cinq sites.

Pour les cinq villes, l'irradiation solaire globale varie proportionnellement au temps au début de la journée pour diminuer ensuite en fin de journée, c.-à-d. elle augmente dès le lever du soleil pour atteindre un maximum à midi solaire avant de décroître à nouveau jusqu'à s'annuler à le coucher de soleil, on remarque que la puissance atteint son maximum à $12 \mathrm{H} 00$. Cette variation enregistrée et due à la position du soleil durant la journée. 
On remarque que pour le site Tamanrasset, le rayonnement global est maximal au midi solaire vrai qui dépasse $700\left[\mathrm{~W} / \mathrm{m}^{2}\right]$. Nous avons enregistré la basse valeur de l'irradiation solaire à Biskra, où sa valeur égale à $465\left[\mathrm{~W} / \mathrm{m}^{2}\right]$.

On remarque que dans les cinq courbes l'évolution des rayonnements solaires globaux sont les mêmes, la différence réside uniquement au niveau des pentes des courbes.

\subsection{La température de sortie de l'air}

Il est bien connu dans la plupart des cas que les capteurs solaires plans sont utilisés pour des applications à basses et à moyennes températures [4].

Pour le chauffage d'un local on utilise l'air comme fluide caloporteur. Nous allons présenter les résultats obtenus après le calcule. Les résultats sont les températures du fluide à l'entrée et à la sortie du capteur solaire plan, durant le déroulement des tests en fonction du temps. Les calculs sont faits pour deux valeurs de débit d'air $\left(Q_{v}=20\left[\mathrm{~m}^{3} / \mathrm{h} \cdot \mathrm{m}^{2}\right]\right.$ et $\left.\mathrm{Q}_{\mathrm{v}}=35\left[\mathrm{~m}^{3} / \mathrm{h} \cdot \mathrm{m}^{2}\right]\right)$.

Les figures $(7,8,9,10$ et 11) représentent l'évolution des températures de sortie de l'air. Les résultats sont calculés heure par heure, du lever jusqu'au coucher du soleil.

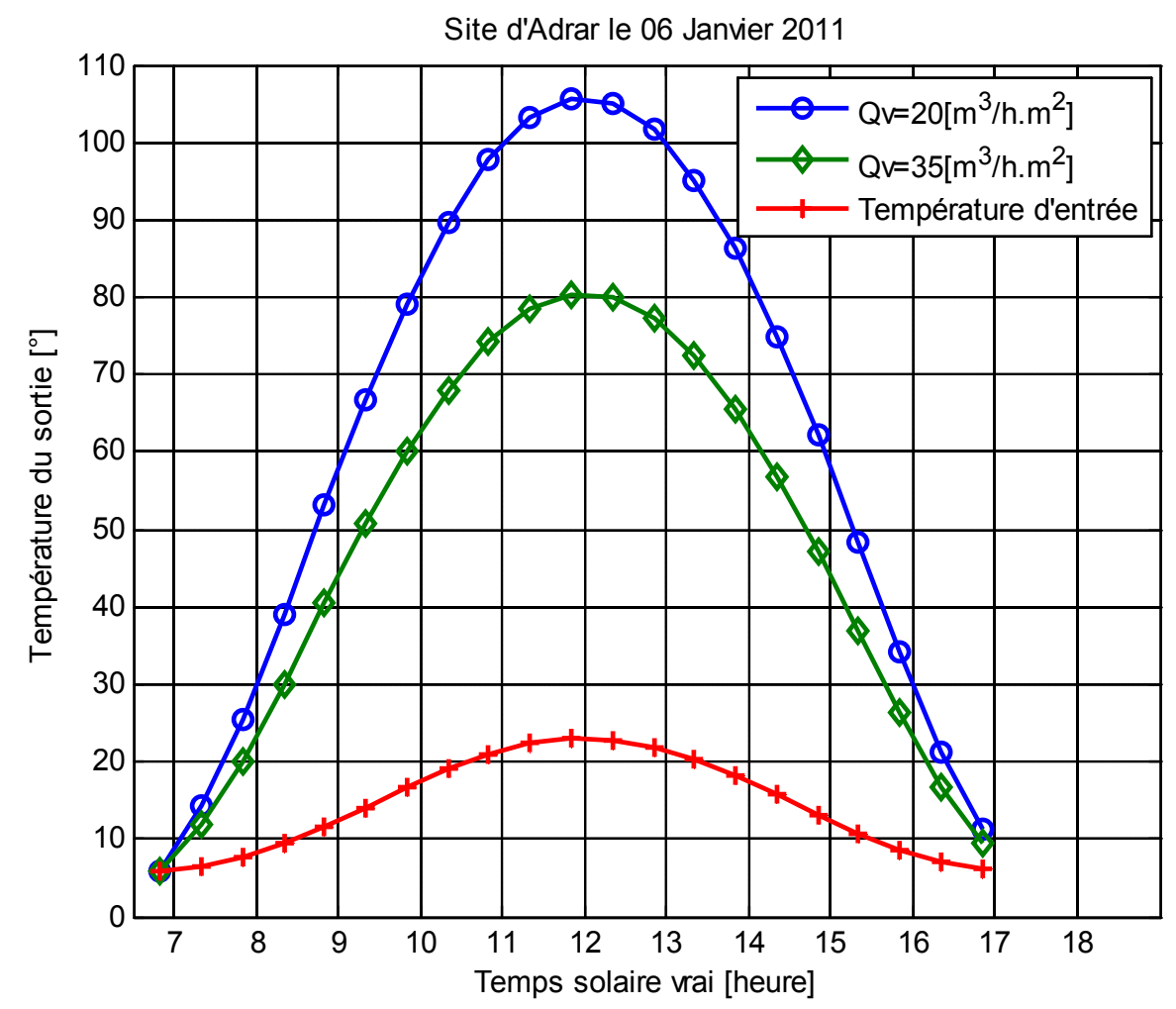

Fig.7. La température de la sortie pour site d'Adrar.

D'après les curbes, on remarque que : 
- l'augmentation de la température du fluide (air) est proportionnelle à la l'augmentation de le rayonnement solaire global, où nous avons remarqué que la valeur maximal de la température de l'air à la sortie de le capteur solaire à $12 \mathrm{H} 00$ étroitement avec la valeur maximale du rauonnement solaire quel que soit le débit volumique de l'air. Après midi, nous observons une diminution progressive de la température de l'air (Ts) jusqu'au coucher du soleil.

- les profils des températures de l'air ont un écart de température entre le point d'entrée et le point de sorti du fluide caloporteur (air); cet écart traduit l'efficacité de l'absorbeur. Par exemple pour le site de Béchar, la différence de température à midi solaire est de $60\left[{ }^{\circ} \mathrm{C}\right]$ avec un débit volumique égale à $35\left[\mathrm{~m}^{3} / \mathrm{h} \cdot \mathrm{m}^{2}\right]$, toute en notant une différence acceptable entre la température d'entré et de sortie de l'air ;

Site de Bechar le 06 Janvier 2011

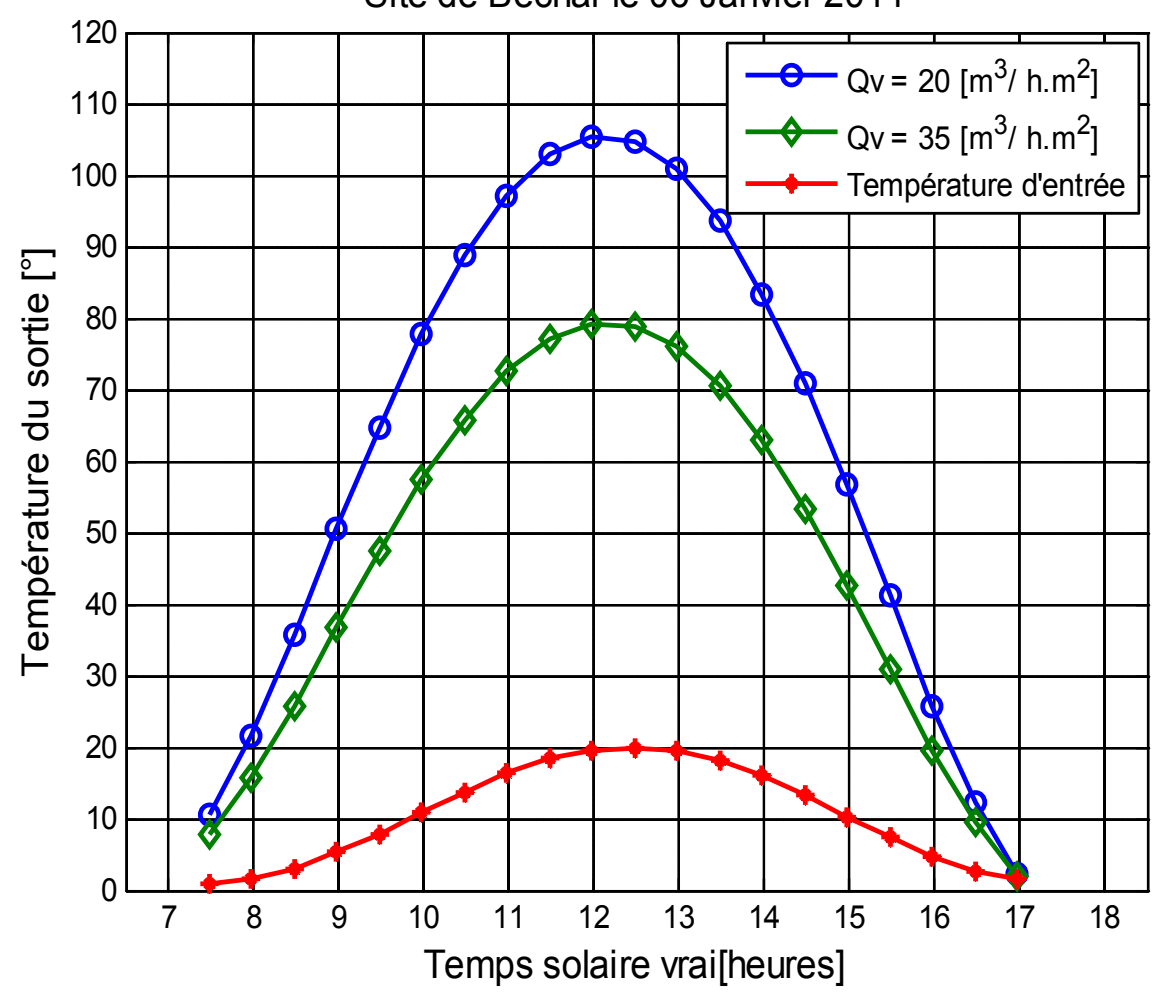

Fig.8. La température de la sortie pour site de Béchar.

- Le débit du fluide caloporteur influe de manière remarquable sur la température de sortie de fluide qui décroissante avec l'augmentation du débit. La température de sortie de l'air est dépendante à la valeur de l'écoulement volumique à l'intérieur de notre prototype ; la dépréciation de la valeur de débit d'air ce qui conduit à l'augmentation de température de l'air à 
la sortie de notre capteur, et vice-versa. Puisque nous parlons sur le débit de l'air, nous profitons de l'occasion pour rappeler l'importance de la choisir la forme de la trajectoire de l'air dans notre collecteur, dont beaucoup de recherches ont montré que l'utilisation des collecteurs solaires avec chicanes accroître leurs performances (l'augmentation de la surface de contact entre l'air et la surface d'absorbation de flux thermique);

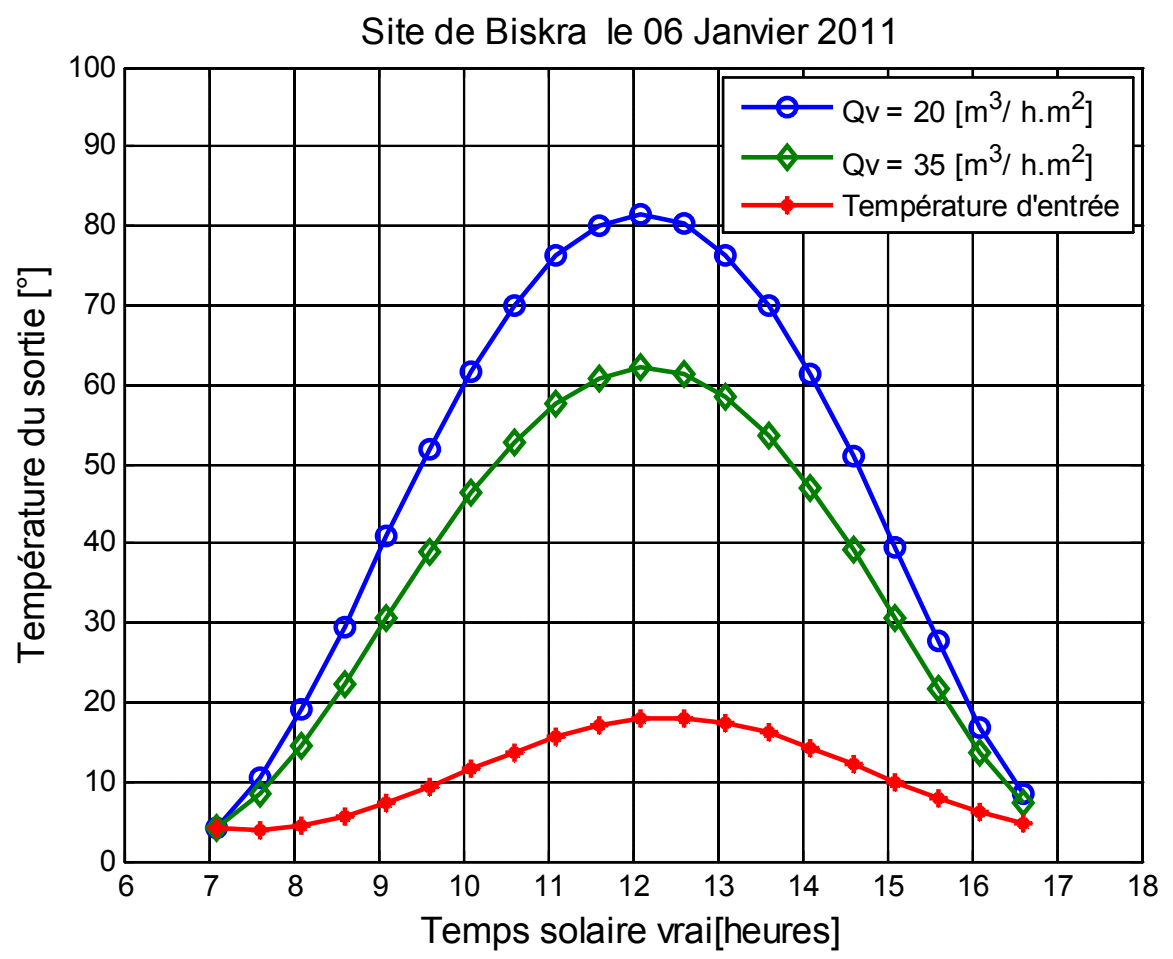

Fig.9. La température de la sortie pour site de Biskra.

- La plus petite valeur des températures à la sortie de le collecteur est enregistrée pour les deux sites Biskra et El Oued, lorsqu'il a dépassé la température dans tous les sites $60^{\circ}$ [C] pour un débit égal à $35\left[\mathrm{~m}^{3} / \mathrm{h} \cdot \mathrm{m}^{2}\right]$. Pour un débit égal à $20\left[\mathrm{~m}^{3} / \mathrm{h} \cdot \mathrm{m}^{2}\right]$, la température a dépassé $80\left[{ }^{\circ} \mathrm{C}\right]$. Les causes de ce diminution n'est pas le manque de le rayonnement solaire, mais la vraie raison est leur paramètres géographiques et climatiques de chaque site (emplacement, l'altitude, la variation de la température d'ambiante, ...etc.) ;

- Indépendamment du lieu, tous les résultats obtenus sont acceptables et encourageants. On observe que, les températures obtenues dans les régions suffisantes pour l'utilisation dans plusieurs axes tels que :

Préchauffage l'air des bâtiments, des écoles, des universités, des hôpitaux, des hôtels, des bureaux, ...etc ; 
Séchage des produits alimentaires ou organiques ;

Chauffage d'un sous-sol ;

Réduction d'humidité dans un local.

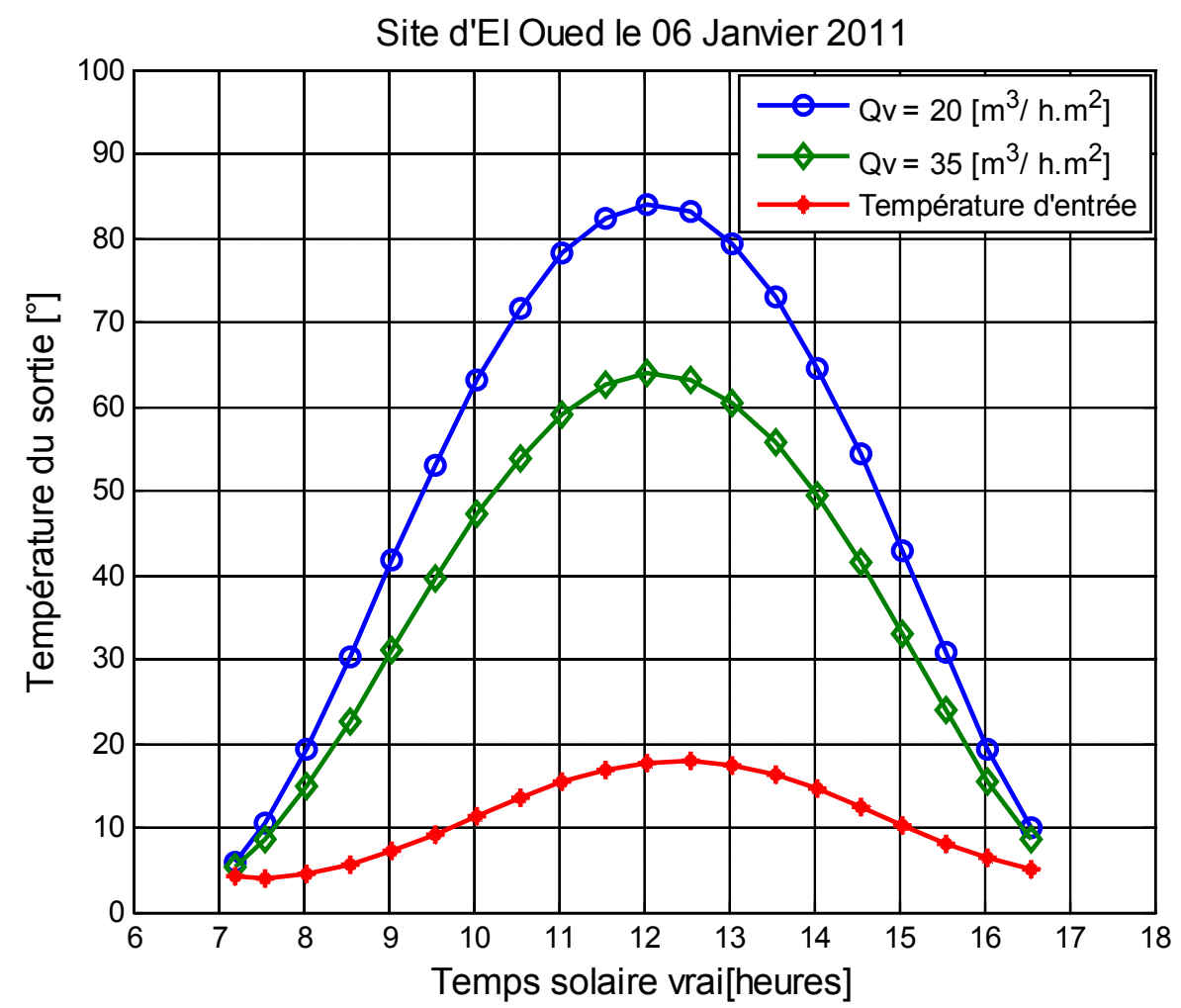

Fig.10. La température de la sortie pour site d'El oued.

- Les cinq régions sont dotées d'un potentiel solaire considérable. Les caractéristiques géographiques et les conditions climatiques influencent également sur le rendement du capteur, l'emplacement des modules en fonction de plusieurs facteurs tels que leurs surface, orientation, inclinaison, type de pose et ombrage, où:

La surface est le facteur responsable sur la détermination de la capacité et de l'énergie (thermique, électrique) qui peut leur fournir avec le capteur ;

L'orientation de notre capteur est vers le sud. La trajectoire du soleil de l'Est à l'Ouest ;

L'inclinaison optimale qui permet de maximiser les gains solaires. Le meilleur rendement du capteur quand l'angle d'inclinaison égal à la latitude de site (l'angle d'inclinaison optimale est perpendiculaire au soleil) ; 
Pour les types de pose, il y a plusieurs types tels qu'au sol, en toiture, en façade et en verrière ;

Concernant l'ombre, est un ennemi juré du capteur, donc, nous devons choisir un endroit qui soit le moins possible soumis aux ombres.

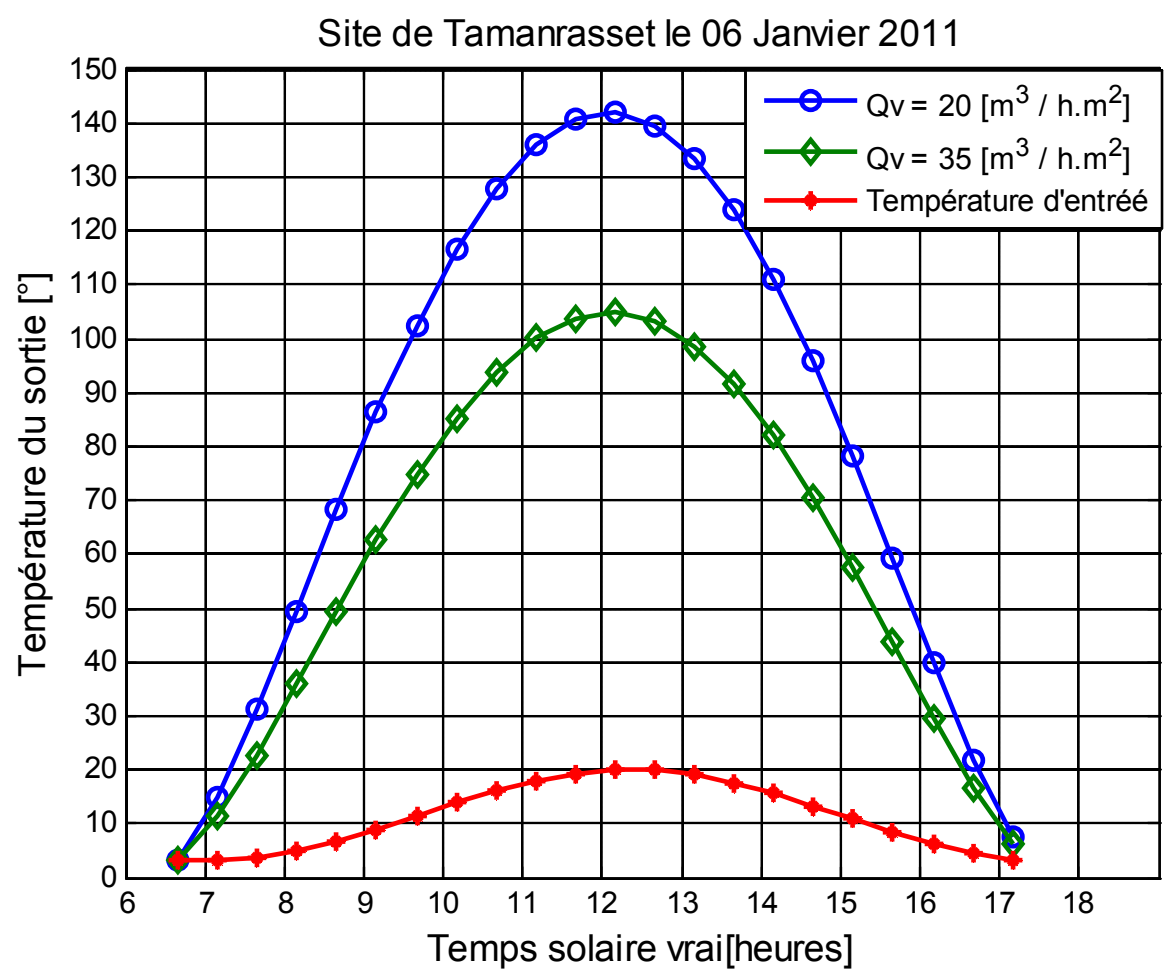

Fig.11. La température de la sortie pour site de Tamanrasset.

Empiriquement, pour augmenter le rendement d'un capteur solaire il faut augmente l'écart de température entre l'entrée et la sortie de l'air, et pour ça il faut :

- choisir la bonne position du capteur, c'est-à-dire le bon choisir l'angle d'incidence ;

- choisir la bonne forme de conduite qui traverse par le fluide (géométrie du capteur).

Donc, les paramètres qui influent sur le rendement sont trop (gisement solaire, position de capteur, géométrie de constructions de capteur, nature de fluide caloporteur, lieu d'entré du fluide au capteur (en haut ou en bas), ...etc.

Finalement, la mise en place de champs d'exploitation de l'énergie solaire (linéaire ou ponctuelle, haute température ou basse température selon le type de collecteur solaire) est le projet de l'avenir de notre pays et de tous les pays du monde. 
Les avantages de notre système chauffe-air solaire sont :

Simplicité d'installation qui nécessite peu d'entretien ;

Réduction des coûts de chauffage ;

Réduction d'émissions de gaz à effet de serre.

\section{CONCLUSION}

Ce travail a entrepris l'étude numérique d'un capteur solaire plan à air. Le rôle d'un capteur solaire est la transformation des rayonnements solaires en énergie thermique extraite par l'air en écoulement dans le capteur. Le rayonnement solaire global après est considéré comme la somme de deux composantes directe et diffus, les formules d'évaluation de ces composantes qui sont présentées dans cet article sont données par le model PERRIN DE BRICHAMBAUT. Le principe de conversion du rayonnement solaire en énergie thermique est basé sur la paroi absorbante qui s'échauffe sous l'effet de l'absorption du rayonnement solaire incident, et l'effet de serre qui consiste à accumuler la chaleur dans un capteur pour la transférer directement ou par l'intermédiaire d'un fluide qui circule sous cette paroi et récupère par convection une partie de cette énergie absorbée et subit une élévation de température $(\Delta \mathrm{T})$ à la traversée du capteur. D'après cette étude, nous pouvons dire que :

- Le Sahara algérien trop large et leur situation géographique permet de bénéficier d'un potentiel solaire très important.

- Le rôle de l'altitude est doublement favorable parce que d'une part, le rayonnement est plus fort à la latitude et egalement à la date, et d'autre part la densité d'air est plus faible, et ceci, pour les deux raisons, réchauffe plus qu'à une altitude plus faible.

- L'augmentation du débit du fluide caloporteur permet de diminuer sa température de sortie et en même temps d'augmenter le rendement du capteur.

\section{REFERENCES}

[1] KALOGIROU S A. Solar Energy Engineering: Processes and Systems. (1st ed), Academic Press, 2009, pp. 7- 42.

[2] RABL A. Active solar collectors and their applications. NewYork Oxford, OXFORD UNIVERSITY PRESS, 1985, pp. 3- 25. 
[3] GHODBANE M, BOUMEDDANE B, LARGOT S, HENIAT N. Etude optique et thermique d'un concentrateur cylindro-parabolique en site d'Alger, Algérie. presented at IXth International Congress on Renewable Energy and the Environment, Djerba, Tunisie, 18-20 March 2015.

[4] GHODBANE M, BOUMEDDANE B, LARGOT S, HENIAT N. Simulation Numérique d'un Concentrateur Cylindro-Parabolique en El Oued, Algérie. International Journal of Scientific Research \& Engineering Technology (IJSET), 3, 2015, pp. 68-74.

[5] GOSWAMI D Y. Energy Conversion: Solar Energy Resources (Chapter 5). Taylor \& Francis Group, LLC, 2007, pp. 1- 9.

[6] SEN Z. Flat Plate Collectors: Solar Energy Fundamentals and Modeling Techniques: Atmosphere, Environment, Climate Change and Renewable Energy. Springer, 2008, pp. 246249.

[7] JANNOT Y. Thermique solaire : Les capteurs solaires plans. Mars 2011, pp. 21-37.

[8] DUfFIE J. A, WILliAM A B. Solar Engineering of Thermal Processes. (4th ed), John Wiley \& Sons, Inc, 2013, pp. 101, 238.

[9] ROMDHANE B S. Rayonnement solaire incident sur les différentes parois d'un bâtiment en Tunisie en fonction de l'heure et du mois. Site de web: http://solairebattunisie.voila.net/index.html, 20/11/2006.

[10] JANNOT Y. Thermique solaire. Octobre 2003.

[11] AHMED-ZAID A, Messaoudi A, Abenne A, Le Ray M, Desmons J, Abed B. Experimental study of thermal performance improvement of a solar air flat plate collector through the use of obstacles: application for the drying of 'Yellow Onion'. International Journal of Energy Research, 23 (12), 1999, pp. 1083-1099.

doi: 10.1002/(SICI)1099-114X(19991010)23:12<1083::AID-ER498>3.0.CO;2-Y

[12] KARIM Md, HAWLADER M N A. Performance investigation of flat plate, vcorrugated and finned air collectors. Energy, 31, 2006, pp. 452- 470.

doi:10.1016/j.energy.2005.03.007

[13] KOYUNCU T. Performance of various designs of solar air heaters for crop drying applications. Renewable energy, 31 (7), 2006, pp. 1073-1088. 
doi:10.1016/j.renene.2005.05.017

[14] MOUMMI N, MOUMMI A, AOUES K, MAHBOUB C, SABRI Y A. Systematic forecasts of solar collector's performance in various sites of different climates in Algeria. International Journal of Sustainable Energy, 29, 2010, pp. 142-150.

doi: 10.1080/14786460903556139

[15] AKPINAR E K, KOÇYIGIT F. Experimental investigation of thermal performance of solar air heater having different obstacles on absorber plates. International Communications in Heat and Mass Transfer, 37, 2010, pp. 416-421.

doi:10.1016/j.icheatmasstransfer.2009.11.007

[16] MOUMMI N. Cours des énergies renouvelables pour deuxième année de Master. Université de Mohamed KHIDER, Biskra, Algérie, 2011.

[17] WEATHER UNDERGROUND. Météorologie Historique. Site de web: http://www.wunderground.com/, 06/01/2011.

[18] MINISTERE DE L'ENERGIE. Potentiels National des Energies Renouvelables. Site de web : http://www.energy.gov.dz/francais/index.php?page=potentiels-national-des-energiesrenouvelables, 03/11/2015.

[19] YAICHE M R, BOUHANIK A. Atlas solaire algérien. Portail des Energies Renouvelables, Centre de Développement des Energies Renouvelables, 2002, pp. 16, 28.

[20] BESSEMOULIN P, OLIVIERI J. LE RAYONNEMENT SOLAIRE ET SA COMPOSANTE ULTRAVIOLETTE. La Météorologie, 8, septembre 2000, pp. 42-59.

[21] WIKIPEDIA. Solar irradiance. Site de web: https://en.wikipedia.org/wiki/Solar_irradiance, 19/08/2015.

\section{How to cite this article:}

Ghodbane M, Boumeddane B, Moummi N, Largot S and Berkane H. Study and numerical simulation of solar system for air heating. J. Fundam. Appl. Sci., 2016, 8(1), 41-60. 